\title{
ANOMALIA MEGADOLICOBASILAR
}

\author{
A PROPOSITO DE 5 CASOS DIAGNOSTICAdOS ANGIOGRAFICAMENTE
}

\author{
WALTER C. PEREIRA * \\ VALFREDO J. NEVES * \\ JOSE ZACLIS **" \\ NELIO G. BARROS ** \\ CELSO FERREIRA "*** \\ YARA RODRIGUES ***
}

Anomalias do calibre e/ou do comprimento da artéria basilar têm sido infrequentemente registradas na literatura, não obstante a grande variedade de manifestações neurológicas a elas atribuidas. A terminologia adotada para designar tais anomalias é diversa, não existindo expressão alguma que as defina de maneira perfeita. Dandy (cit. por Adams e col. 1) descreveu-as como) artérias em $S$; o termo ectasia tem sido utilizado nos casos em que ocorre hidrocéfalo por compressão do terceiro ventículo 4, 6, 7; outras expressĩes também encontradas na literatura são aneurisma e dilataçũo fusiforme da basilar 11. O termo megadolicobasilar foi pela primeira vez empregado por Boeri e Passerini 5, visando a descrever artéria basilar anormalmente dilatada, alongada e sinuosa, sendo desde então adotado pela maioria dos autores. Segundo alguns, esta anomalia está associada com frequência a alterações da mesma natureza das artérias carótidas ou de outros vasos $5,14$.

O presente trabalho tem por finalidade a discussão de 5 casos de megadolicobasilar, que tivemus ocasião de diagnosticar em nosso) Serviço nos últimos três anos, no qual analisaremos alguns dos aspectos clínicos relacionados a estas anomalias vasculares, salientando a importância do estudo angiográfico encefálico, único exame capaz de estabelecer seu diagnóstico, que se presta à confusão com várias outras afeç̧ões, inclusive de caráter expansivo.

\section{OBSERVAÇÕES}

Caso 1 - A.D.E., sexo masculino, cor branca, 60 anos de idade. registro 41.9:36. Sensação de tontura. acompanhada de queda da pálpebra direita e. três meses depois. perda súbita de força nos membros esquerdos. Paciente com hipertensão arterial de longa duraçăo, nåo tomando đrogas hipotensoras de maneira constante. Exame clinico-neurologico - Pressão arterial $220 \times 120 \mathrm{~mm}$ de $\mathrm{Hg}$ : pulso regular e rítmico. 90 batimentos/min. Consciente e orientado: paralisia completa do nervo motor ocula" comum direito; hemiplegia esquerda total e proporcionada: sinais piramidais de libe-

Trabalho realizado no Hospital Săo Jaaquim da Real e Benemêrita Sociedade Portuguesa de Beneficência de Săo Paulo: * Chefe do Serviço de Neurocirurgia: ** Neurocirurgiăo Assistente: *** Neurorradiologistas: *** Médicos Residentes. 
ração generalizados predominando nos membros esquerdos: fundos oculares mostrando espasmo arteriolar e arteriolosclerose $\left(\begin{array}{ll}\mathbf{H}_{2} & \mathbf{A}_{\mathbf{2}}\end{array}\right)$.

Exames complementares - Craniogramas simples normais. Eletrencefalograma normal. Liquido cefalorraqueano (SOD); células e proteínas normais, reação de Pandy opalesente. Eletrocardiograma: sobrecarga das câmaras esquerdas e alterações difusas da repolarização ventricular. Pneumencefalograma normal. Angiografia carotídea bilxteral: artérias tortuosas e com calibre irregular decorrentes de aterosclerose: angiografia vértebro-basilar: artéria basilar anormalmente longa e calibrosa, descrevendo desvio acentuado da esquerda para a direita, na incidência anteroposterior. e grande curvatura de convexidade posterior. na incidência lateral (fig. 1.1).

Evolução - Durante as 4 semanas em que permaneceu internado, o paciente recebeu medicação anti-adesiva plaquetária (AAS e dipiradamol) e hipotensora (metildopa). tendo alta com melhora discreta do deficit motor dos membros esquerdos.

Caso 2 - E.A., sexo masculino, cor branca, 64 anos de idade, registro 32.408. Tontura intensa, acompanhada de náuseas e vômitos, com inf́cio súbito 24 horas antes da internação. Era hipertenso há cerca de 10 anos, não se tratando adequadamente. Exame clinico-neurológico - Pressão arterial $180 \times 110 \mathrm{~mm}$ de $\mathrm{Hg}$ : pulso regular e rítmico, 60 batimentos/min. Consciente e orientado: distasia e disbasia: marcha com tendência à queda para a esquerda: dismetria e decomposiçăo de movimentos com predominio nos membros esquerdos: fundos oculares mostrando espasmo arteriolar e arteriolosclerose $\left(\begin{array}{ll}H_{1} & A_{2}\end{array}\right)$.

Exames complementares - Craniogramas simples normais. Eletrencefalograma: desorganização difusa e alentecimento da atividade elétrica cerebral. Liquido celaforraqueano: 5 células, 2.100 hemácias degeneradas $(53,6 \%$ de oxi-hemoglobina e $46,4 \%$ de bilirrubinas), $40 \mathrm{mg} / 100 \mathrm{ml}$ de proteinas. Eletrocardiograma: bradi-arritmia sinusal e sobrecarga ventricular esquerda. Angiografia carotídea bilateral: artérias tortuosas e com calibre irregular. Angiografia vértebro-basilar: artéria basilar anormalmente longa e calibrosa apresentando trajeto sinuoso (fig. 1.2).

Evolução - Logo após o estudo angiográfico o paciente queixou-se de queda bilateral acentuada da acuidade visual, não tendo sido observadas modificações apreciáveis dos fundos oculares. Recebeu medicação vasodilatalora (cloridrato de papaverina), anti-adesiva plaquetária (AAS e dipiradamol) e hipotensora (metildopa), tendo alta cerca de um més depois com melhora nitida do quadro cerebelar, mas ainda apresentando redução da acuidade visual.

Caso 3 - M.P.F.P., sexo feminino, cor branca, 56 anos de idade, registro 58.410. Dois anos antes da internação tivera subitamente cefaléia e vômitos; desde então apresentava tremores nos 4 membros, principalmente nos superiores, e dificuldade para articular a palavra. Um ano antes desse quadro a paciente sofrera enfarte do miocárdio, sendo hipertensa há vários anos, não tomando medicação de maneira constante. Exame clínico-neurologico - Pressão arterial $180 \times 100 \mathrm{~mm}$ de $\mathrm{Hg}$; pulso irregular e arritmico, 100 batimentos/min. Consciente e orientada; sindrome cerebelar global intensa.

Exames complementares - Craniogramas simples normais. Eletrencefalograma normal. Liquido cefalorraqueano (SOD): células normais, $40 \mathrm{mg} / 100 \mathrm{ml}$ de proteinas reação de Pandy opalescente. Eletrocardiograma: zona inativa na parede anterior e extrassístoles ventriculares raras. Pneumencefalograma normal. Angiografia carotidea bilateral: segmentos intracranianos de ambas as carótidas internas mais calibrosos que os cervicais; artérias tortuosas com sinais de aterosclerose. Angiografia vértebro-basilar: artéria basilar anormalmente longa e calibrosa, apresentando-se desviala da direita para a esquerda, na incidência anteroposterior, e com curvatura de convexidade anterior. na incidência lateral (fig. 1.3).

Evolução - A paciente recebeu medicação hipotensora (metildopa) e anti-adesiva plaquetária (AAS e dipiradamol), tendo alta três semanas depois da internação com o quadro neurológico inalterado.

Caso 4 - I.C.R.A., sexo feminino, cor branca, 46 anos de idade, registro 89.206. Seis horas antes da internação a paciente apresentara subitamente tontura, vơmito, 
visão dupla, diminuição de força nos membros esquerdos e, a seguir ficou sonolenta. Apesar de ser hipertensa há muitos anos, somente iniciou o uso de drogas hipotensoras 6 meses antes do quadro, que motivou sua internação. Exame clinico-neurologico Pressão arterial $220 \times 110 \mathrm{~mm}$ de $\mathrm{Hg}$; pulso regular. 88 batimentos/min. Sonolenta: desvio conjugado do olhar para a direita; hemiparesia esquerda completa e proporcionada.

Exames complementares - Craniogramas simples normais. Eletrencefalogrania: desorganização difusa e alentecimento da atividade elétrica cerebral. Angiografia vértebro-basilar: acentuados alongamento e aumento de calibre da artéria basilar, que apresenta cm sua porçăo inicial grande dilatação aneurismática fusiforme; esta artéria diri'̧i-se da esquerda para a direita, descrevendo curva fechada de convexidade direita: a artéria cerebral posterior năo ê visibilizada, não sendo possível, porêm, ufırmar-se se está ocluída por ter sỉo suspenso o exam? antes do estudo das artérias carótidas (fig. 1.4).

Evolucăo - Com medicação hipotensora (metildopa) e anti-adesiva plaquetária (AAS e dipiradamol) houve de início milhora do quadro neurjógico da paciente. Dois dias depois da internação, porém, ocorreu novo episódio de homiparesia, agora localizada à direita. Cinco dias depois a loente qujix su-se de cefuléiu intensa. de instalação sūbita apresentando logo a seguir coma profunds e postura em descerebração. O líquido cefalorraqueano examinado nesta ocasiăo mosirou-se fortemente hemorrágico e hipcrtcnso, falecendo a doente três dias após este novo icto.

Caso 5 - F.C.R., sexo masculino, cor branca, 46 anos de idade, registro 101.544. Cefaléia intensi. tontura, vômitos e turvaçăo da visão dois dias antes da internação: no dia seguinte dificuldade para articular a palavra: foi consultado um clínico que diagnosticou crise hipertensiva. Exam ̌ cünico-neurológico - Pressão arterial $180 \times 120 \mathrm{~mm}$ de $\mathrm{Hg}$; pulso rcgular, 80 batimentos/min. Consciente; discreta sonolencia; sem deficits sensitivo-motores: ausência de siñis de irritação meningea: fundos ocularcs mostrando (spasmo arteriolar intenso e arteriolosclerose $\left(\mathrm{H}_{3} \mathrm{~A}_{2}\right)$.

Exames complcmentares - Craniogramas simplcs normais. Eletrencefaiogramx normal. Eletrocardiograma: zona elctricamente inativa na parede diafragmática. Liquido cefalorraqueano: 1.mpido e incolor. ausência de cẹlulas, $58 \mathrm{mg} / 100 \mathrm{ml}$ de proteinas, reações de Pandy e 'Takat:ג-Ard positivas. Esiudo angiografico carotideo bilateral e vêricbro-basilar por catcterismo da artéria femoral: artéria basilar muito longa e calibrosa, apresentan do desvio moderado da direita para a esquerda, sob a forma de curvatura leve de convexidade esquerda; na incidência lateral observa-se acentuada curvatura de sua porção terminal de convexidade anterior (fig. 1.5): as artérias dos territórios carotídeos apresentam calibre irregular e sinais acentuados de aterosclerose.

Evoluçao - O paciente admitido com exams neurológico normal, foi aprcsentando. de maneiru progressiva, quadro de hemiplegia alterna, com paralisia facial periférica esquerda e deficit motor nos membros direitos (sindrome de Millard-Gubler), que se instalou completamenie no quarto dia de internaçio. Apesar de se instituir medicação hipotensora (metildopa), anticoagulante (heparina) e anti-edema cerebral (dexametasona), o estado neurológico do doente foi piorando progressivamente, vin lo a falecer em coma protundo 14 dias depois.

\section{COMENTAFIOS}

Várias manifestaçōes neurulógicas isoladas ou associadas entre si têm sido relacionadas à presença de megadolicobasilares, predominando na literatura o relato de comprometimento de nervos cranianos, hidrocéfalo de pressão normal e síndromes do ângulo pontocerebelar e do tronco cerebral.

$\mathrm{O}$ acometimento de nervos cranianos é geralmente atribuído à compressão exercida diretamente pela artéria basilar ectásica, manifestando-se quase sempre por sinais e sintomas de natureza irritativa. Os nervos mais comumente compro- 

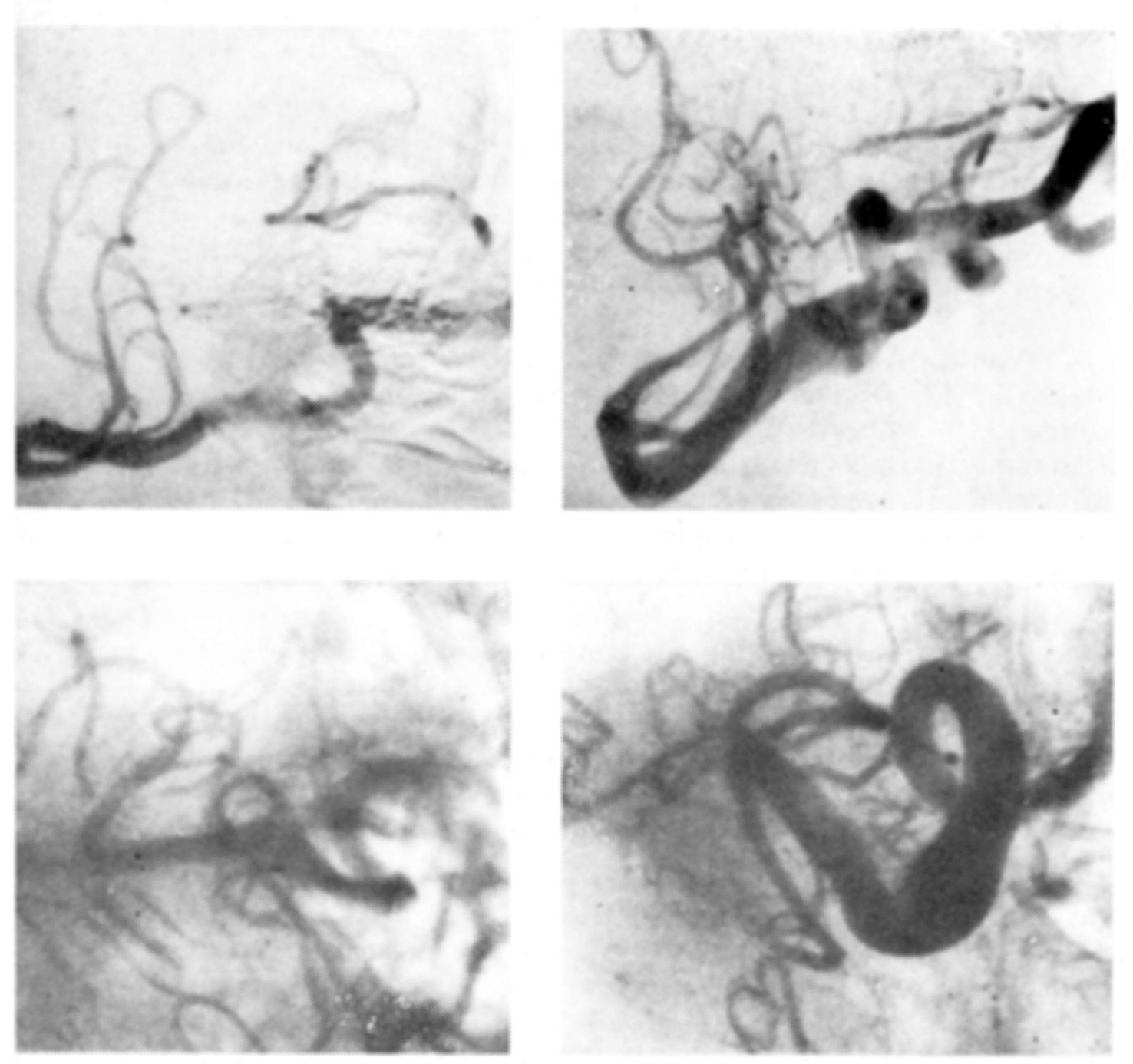

N
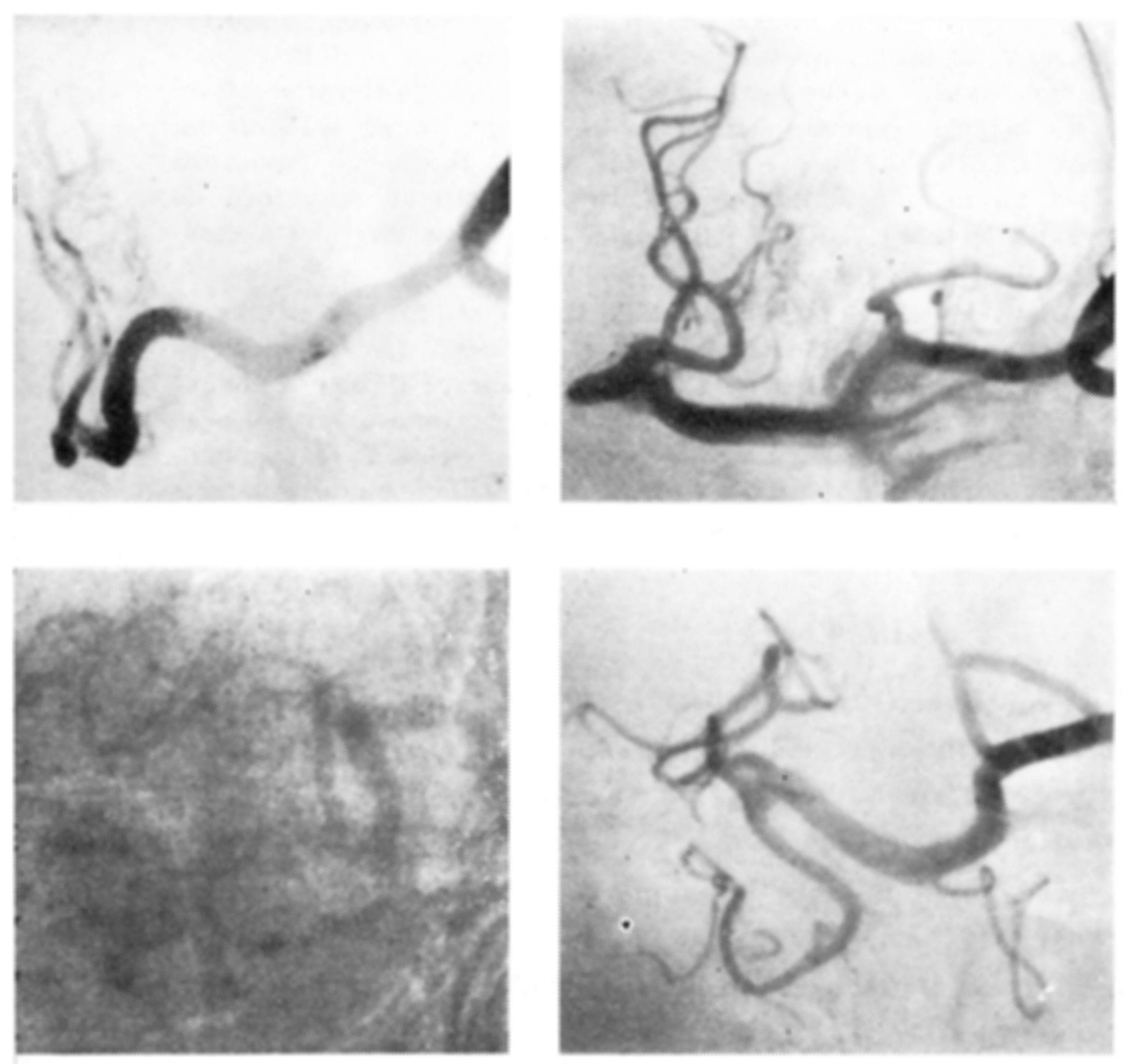

$m$

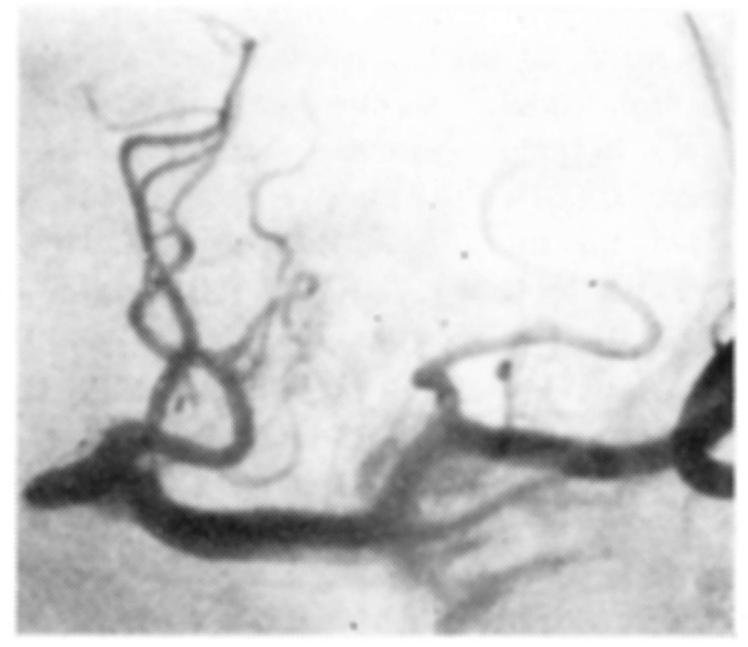

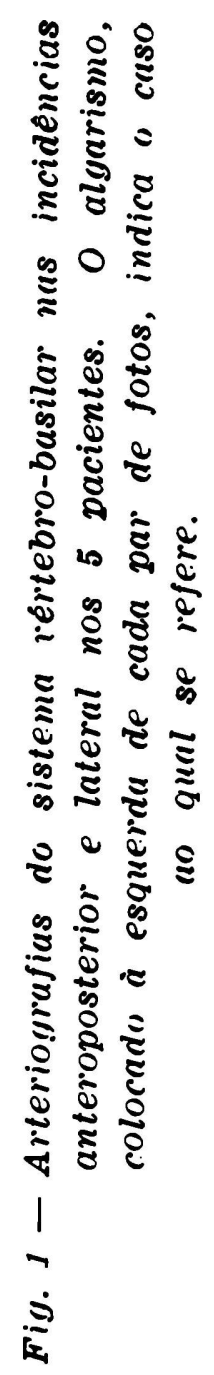
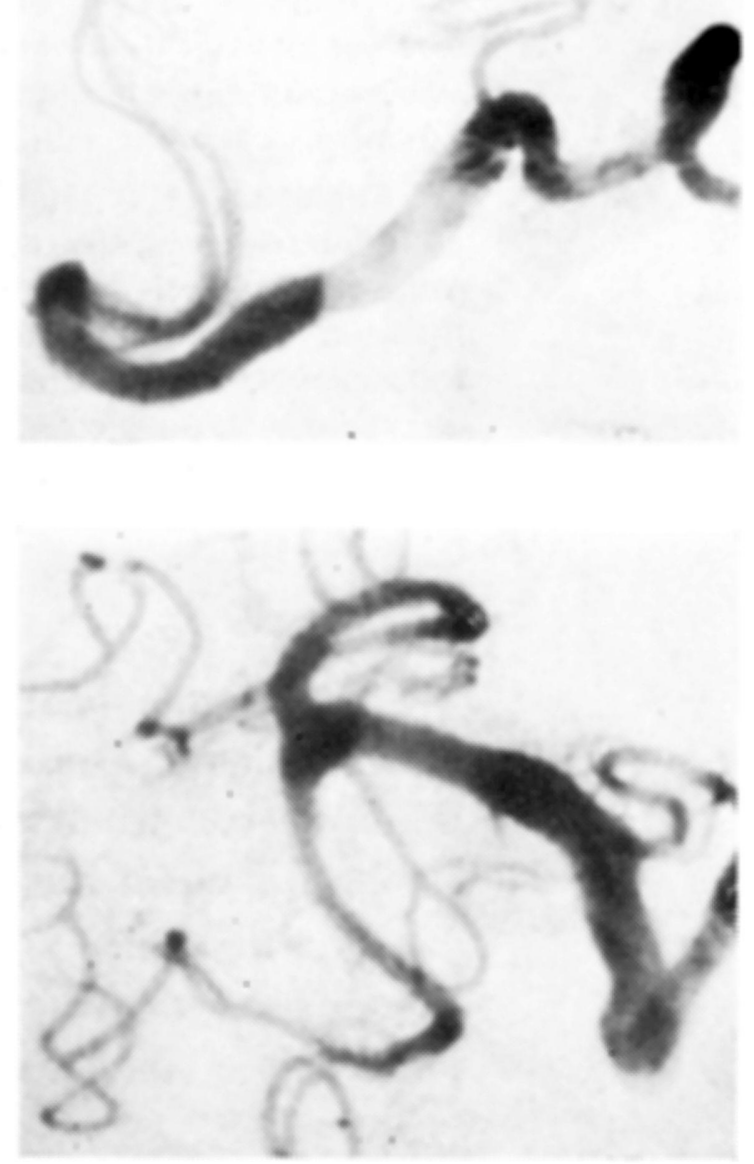

ת 
metidos são, por ordem de freqüência, o trigêmeo, o facial e o motor ocular comum. Dandy (cit. Janneta 15 ) encontrou, entre 215 pacientes com neuralgia essencial do trigêmeo, $6(2,8 \%)$ em que esta era causada por compressão da raiz sensitiva do nervo por aneurisma da artéria basilar. Por este motivo, Janneta ${ }^{15}$, entre outros, preconiza a prática sistemática de angiografia vértebro-basilar em doentes com neuralgia do trigêmeo, atribuindo a fatores compressivos localizados na fossa craniana posterior papel de relevância na etiopatogenia desta afecção, tida comumente como essencial. $O$ espasmo facial, na maior parte das vezes também rotulado como criptogenético, foi associado a muitas causas, destacando-se entre estas a compressão do sétimo nervo por artéria basilar anormalmente calibrosa e sinuosa $3,6,7,11,12,19$. Nos casos de megadolicobasilar observados por Boeri e Passerini 5 o nervo motor ocular comum foi o mais frequentemente afetado. Segundo este autores seu comprometimento é, em geral, parcial, sendo acometido principalmente a motricidade ocular intrínseca, a ela associando-se paresia dos músculos elevador da pálpebra, reto superior e reto medial; notaram ainda que o nervo lesado era o localizado no lado correspondente à convexidade descrita pela megadolicobasilar, afirmando, porisso, ser a compressão do nervo em seu trajeto infratentorial a causa da sintomatologia. Outros autores, contudo, não confirmam a elevada incidência de acometimento do terceiro nervo em casos de megadolicobasilar 7.

Hidrocéfalo de pressão normal também tem sido relacionado à presença de megadolicobasilar 1.4.8.9. Breig e col. ${ }^{4}$ e Ekbom e col. ${ }^{4} . "$ registraram 9 casos dessa sindrome, em que o terceiro ventriculo estava moderada ou intensamente deformado por artéria basilar ectásica. Estes autores atribuem à transmissão das fortes pulsações da artéria dilatada ao sistema ventricular o desenvolvimento progressivo do hidrocéfalo que, em sua experiência, sempre se manifestou em indivíduos hipertensos e após a quarta década da vida. Relacionam ainda à compressão dos corpos mamilares os distúrbios de memória comumente presentes em seus doentes.

Sindromes atípicas do ângulo pontocerebelar causadas por grande ectasia da artéria basilar têm sido ocasionalmente registradas na literatura $3,11,19$. Nestes casos podem estar associados hipertensão intracraniana, quadro cerebelar axial e/ou apendicular, deficits motores e/ou sensitivos dimidiados e comprometimento mais ou menos extenso de nervos cranianos (geralmente do quinto ao oitavo). Do ponto de vista do diagnóstico diferencial com tumores sensu strictu, o quadro neurológico nestes casos apresenta-se geralmente com início e evolução de tipo vascular, situando-se a idade dos pacientes acima da quinta década e o estudo radiológico do crânio não costuma evidenciar as lesões ósseas características das neoplasias desta região. Por outro lado, contudo, pode ocorrer dissociação proteíno-citológica acentuada no líquido cefalorraqueano e o pneumencefalograma, assim como a ventriculografia gasosa ou com substância contrastante positiva podem revelar a presença de massa expansiva pontocerebelar. Somente a angiografia vértebro-basilar fornece elementos decisivos para o diagnóstico diferencial nestes casos, sendo, portanto, imprescindível sua prática. 
Teoricamente todas as sindromes vasculares do tronco cerebral podem estar presentes em casos de megadolicobasilar `. Foram descritas na literatura sindromes de hemiplegia alterna, oftalmoplegia internuclear, alucinações visuais, mioclonias do véu do paladar, hemiparesia ou paraparesia espásticas, hemianopsia homônima e deterioração psíquica progressiva 5, 10, 17. Estas manifestações podem ser transitórias, recidivantes ou de caráter alternante, em casos de embolia partida de trombos murais localizados na artéria basilar afetada, ou definitivas, quando decorrem de trombose com oclusão total de um ou mais de seus ramos.

Outras manifestações têm sido descritas em pacientes com megadolicobasilar, tais como enxaqueca, cefaléia occipital paroxística, presença de sopro ou frêmito mais ou menos extensamente irradiado da região occipital 2.

O diagnóstico de megadolicobasilar é raramente feito, sendo excassas as publicações sobre o assunto. Isto se deve, provavelmente, ao fato dessa afecção incidir quase sempre em indivíduos relativamente idosos, sendo o caso rotulado como acidente vascular cerebral comum, não se praticando estudo angiográfico de rotina. A associação frequente dessa anomalia com hipertensão arterial e aterosclerose faz com que seja geralmente considerada como decorrente da involução senil das paredes arteriais, tendo sido demonstrada atrofia das camadas muscular e elástica dos vasos afetados, causando sua dilatação, tortuosidade e alongamento 5, 18. No entanto, a descrição de um (aso ocorrido em adolescente 13 , bem como a associação com malformações congênitas, tais como angioma arteriovenoso e malformação do circulo arterial do encéfalo 14,impressão basilar e anastomose occipitovertebral 7, tornam plausivel que, em alguns pacientes, a megadolicobasilar possa ter caráter malformativo.

Todos os casos de megadolicobasilar por nós observados ocorreram em doentes com mais de 46 anos de idade, estando associada à hipertensão arterial e aterosclerose, fatos estes concordantes com os relatados na maioria dos trabalhos sobre o assunto. Da mesma forma a variabilidade do quadro neurológico encontrado nesses doentes confirma o carảter proteiforme com que esta afecção se manifesta. Assim, em dois pacientes (casos 1 e 5) instalou-se hemiplegia alterna (Weber e Millard-Gubler, respectivamente); em outros dois (casos 2 e 3) observou-se quadro cerebelar e em uma doente (caso 4), hemiplegia esquerda e direita sucessivamente. Além dessas manifestações, em dois doentes (casos 2 e 4) ocorreu hemorragia subaracnóidea, no segundo seguida de coma profundo e descerebração.

Em todos os pacientes a instalação do quadro foi de tipo ictal, sugerindo natureza vascular arterial do processo e, pelos sintomas e sinais observados, sua localização no território vértebro-basilar.

Quanto aos exames complementares, observamos que o liquido cefalorraqueano, examinado em todos os pacientes, revelou-se hemorrágico em dois (casos 2 e 4), estando a proteina nitidamente elevada apenas em um (caso 5). O eletrencefalograma, também praticado em todos os doentes, mostrou-se alterado apenas em dois (casos 2 e 4), nos quais revelou desorganização difusa e alentecimento da atividade elétrica cerebral. O eletrocardiograma, feito em 4 doentes, 
demonstrou enfarte pregresso em dois (casos 3 e 5) e distúrbios relacionados à hipertensão arterial e aterosclerose nos outros dois (casos 1 e 2). O pneumencefalograma, praticado somente em dois pacientes (casos 1 e 3), mostrou-se normal em ambos. O estudo angiográfico carotídeo bilateral, realizado em todos os doentes exceto um (caso 3), revelou sinais de aterosclerose generalizada das artérias cerebrais, mostrando apenas em um (caso 3) concomitância de megadolicocarótida de ambos os lados. A angiografia do sistema vértebro-basilar foi o exame que tornou possível o diagnóstico em todos os casos.

\section{RESUMO}

Após breves considerações sobre a sintomatologia e etiopatogenia da megadolicobasilar, são apresentados 5 casos desta anomalia vascular. Concordando com a maioria dos trabalhos sobre 0 assunto, a idade dos doentes desta série situava-se entre a quarta e sexta décadas da vida, apresentando todos hipertensão arterial de longa duração e aterosclerose avançada; o quadro neurológico deste doentes mostrou-se variável, tendo em comum o fato de ter se iniciado abruptamente. $O$ estudo angiográfico do sistema vértebro-basilar foi o exame que possibilitou o diagnóstico em todos os casos.

\section{SUMMARY}

Megadolichobasilar anomaly: report of 5 cases with angiographic study

After brief considerations on the symptomatology and ethiopathogeny of megadolichobasilar, five cases of this vascular anomaly are presented. Agreeing with the majority of the papers on the subject, the age of the patients was between the fourth and the sixth decades, all of them presenting arterial hypertension of long duration and advanced atherosclerosis; the neurological findings were varied, having in common the fact of an abrupt (ictal) beggining. The angiographic study of the vertebrobasilar system was basic in the diagnosis in every case.

\section{REFERENCIAS}

1. ADAMS, R.D.: FISCHER. C.M. : HAKIM, S.: OJEMANN, R.G. \& SWEET, W.H. - Symptomatic bccult hydrocephalus with "normal" cerebrospinal fluid pressure: a treatable syndrome. New Engl. J. Med. 273:117, 1965.

2. ANDR J.M. - Les Dysplasies Vasculaires Systematisées. Expansion Scientifique Française, Paris, 1973, pp. 103-116.

3. BINGAS, B. \& COTSON, S. - Cerebello-pontine angle: syndrome with uncommon aethiology. Neuroradiology $3: 165,1972$.

4. BREIG, A.; EKBOM, K.; GREITZ, T. \& KUGELBERG, E. - Hydrocephalus due to elongated basilar artery: a new clinic-radiological syndrome. Lancet i:874, 1967.

5. BOERI, R. \& PASSERINI. A. - The megadolichobasilar anomaly J. neurol. Sci. $1: 475,1964$.

6. CARELla, A.: CARUSO, G. \& LAMBerTi, P. - Hemifacial spasm due to elongation of the distal segment of the vertebral artery: report of two cases. Neuroradiology 6:233, 1973. 
7. Dehaene, I.; PATTYN, G. \& CALLIAUW, L. - Megadolichobasilar anomaly. basilar impression and occipito-vertebral anastomosis. Clin. Neurol. Neurosurg. 78:131, 1975.

8. EKBOM, K.; GREITZ. T.; KALMER, M.; LOPEZ, J. \& OTTOSSON, S. - Cerebrospinal fluid pulsations in occult hydrocephalus due to ectasia of basilar artery. Acta Neurochir. (Viena) 20:1, 1969.

9. EKBOM, K.; GREITZ, T. \& KUGELBERG, E. - -Hydrocephalus due to ectasia of the basilar artery. J. neurol Sci. 8:465, 1969.

10. FRANCK. G.; RESNIK, M. \& THIBAUT, A. - tule clinique et anatomique d'un cas de clonies vêlo-pharyngo-laryngées secondaires à anévrysme fusiforme athérosclếreux du tronc vertébro-basilaire. Rev. Neurol. (Paris) 113:56, 1965.

11. GERAUD, J.; RASCOL, A.; BeS, A.; ARBUS, L. \& BENAZET A.M. - Anévrysme fusiforme vertébro-basilaire à symptomatologie pseudo-tumorale: Átude clinique et radiologique. Rev Neurol. (Paris) 110:66, 1964.

12. GIBSON, W.P.R. \& WALLACE, D. - Basilar artery ectasia: an unusual cause of a cerebello-pontine lesion and hemifacial spasm. J. Laring. Otol. 87:721, 1975.

13. GONSETTE, R. \& ANDRE-BALLISAUX, G. - Incidences clinique et radiologique des mega-artéres basilaires. J. Radiol. Eletrol. 45:248, 1964.

14. HEIDRICH, R. \& SCHNEIBER, D. - Arteriovenous angioma, megadolichobasilaris and malformations of the circle of Willys. Psychiat. Neurol. Med. Psychol. (Leipzig) 22:177 1970.

15. JANNETA, P.J. - Arterial compression of trigeminal nerve at the pons in patients with trigeminal neuralgia. J. Neurosurg. 26:159, 1967.

16. KERBER, C.W.; MARGOLIS, M.T. \& NEWTON, T.H. - Tortuous vertebrobasilar system: a cause of cranial nerve signs. Neuroradiology 4:74, 1972.

17. TAKAHASHI, M. - Atlas of Vertebral Angiography. Inaku Shoin, Tokio, 1974.

18. SCHINDLER, E. - A brain stem syndrome caused by megadolichobasilaris. Z. Neurol. $205: 221,1973$.

19. TRIDON, P.; MASINGUE. M.; PICARD, L.; BRIQUELL, F. \& ROLAND, J. Hémispasme facial et mégadolichobasilaire à symptomatologie pseu lo-tumorale. Rev. Otoneuroophtalmol. 43:279, 1971.

Servico de Neurocirurgia - Hospital da Beneficência Portuguesa de såo Paulo -. Rua Maestro Cardim 769, sala 275 - 01323 Săo Paulo, SP - Brasil. 